\title{
ENJEUX ET DÉFIS DES POLITIQUES LOCALES D'ADAPTATION AU CHANGEMENT CLIMATIQUE EN AQUITAINE
}

\author{
Julien Rebotier \\ CNRS - Laboratoire SET (UMR 5603), Institut Claude Laugénie, \\ Domaine universitaire, 64000 Pau - France \\ julien.rebotier@cnrs.fr
}

\begin{abstract}
Résumé : L'adaptation au changement climatique s'impose tant dans les institutions internationales que dans le débat académique et l'action publique, nationale et locale. La mise en place de stratégies d'adaptation locales est en France la marque des lois liées au Grenelle de l'environnement. Dans un contexte de remise en question des rapports entre sociétés et milieux, de montée des incertitudes, et de redéfinition des acteurs légitimes à élaborer et intervenir dans la gestion territoriale, l'analyse de l'application de ces stratégies sur les territoires d'Aquitaine présente trois enseignements majeurs. Elle montre la difficulté de leur appropriation locale, l'existence d'un champ d'opportunités pour les acteurs locaux malgré la difficulté à s'extraite d'un modèle descendant de l'action publique, et l'enjeu pour les sciences sociales de saisir les dimensions sociopolitiques et humaines d'une question environnementale trop souvent réduite aux considérations de sciences dites exactes et à l'incertitude des scénarios de climat futur.
\end{abstract}

Mots-Clés : adaptation, territoires, gouvernance, planification, épistémologie.

Resumen: La adaptación al cambio climático se impone tanto en las instituciones internacionales como en la academia o en la acción pública, nacional y local. La implementación de estrategias locales de adaptación en Francia es una marca de las leyes Grenelle de l'Environnement. Mientras se cuestionan las relaciones entre sociedades y ambiente, mientras se consolidan dudas e incertidumbres, y mientras se redefinen los actores legítimos a la hora de elaborar e intervenir en la gestión territorial, el aná-

Recibido: 16-04-13. Aceptado: 17-10-13. 
lisis de la operacionalización de aquellas estrategias en los territorios de Aquitania presenta tres resultados principales. Subraya la difícil apropiación local de las estrategias, evidencia el abanico de oportunidades que existen para los actores locales a pesar de las dificultades de librarse de un modelo descendiente de acción pública, y hace hincapié en la importancia para las ciencias sociales de conceptualizar la cuestión ambiental a partir de sus dimensiones sociopolíticas y humanas mientras que se reduce a menudo a un enfoque de geociencias y a la incertidumbre de la modelización del clima.

Palabras clave: adaptación, territorios, gobernanza, planificación, epistemología.

\section{L'adaptation au changement climatique : vers une gestion territoriale renouvelée?}

Depuis les années 1970, la question environnementale se structure, s'institutionnalise et amène à interroger tant les politiques publiques que les modèles de développement basés sur la croissance et le progrès (Rist, 1996). Elle en vient même à participer aux recompositions de la modernité (Beck, 2003), poussant individus et sociétés à un effort important de réflexivité (Serres, 1990 ; Morin, 1993). Contemporaine d'une montée en puissance des incertitudes et d'une crise de confiance parmi des acteurs ou en des institutions (comme l'État, le savoir expert ou la science) jusque là légitimes dans l'administration des preuves ou l'initiative de l'action (Theys, 2003), la question environnementale, devenue globale, interroge au tournant du 21e siècle la capacité des sociétés à configurer leurs futurs possibles (Innerarity, 2008). C'est donc une question sociale totale que donne à connaître la question environnementale. C'est notamment le cas aujourd'hui à travers la problématique du changement climatique, à travers les politiques et initiatives qui lui sont liées, ainsi que les opportunités d'innovation qui se présentent en matière de planification et de démocratie locale (Lolive et Soubeyran, 2007 ; Berdoulay et Soubeyran, 2012).

De fait, la littérature a déjà exploré la façon dont le changement climatique, au-delà des dynamiques physiques qui le caractérisent, peut être historicisé et construit comme un objet social, politiquement contingent, et marqué par des formes d'organisation et de fonctionnement sociaux à différentes échelles (Demeritt, 2001). Pour les sciences sociales, ce ne sont pas les dimensions physiques de l'environnement qui sont au cour des préoccupations. Le changement climatique n'a de sens et d'intérêt qu'à travers les logiques et principes sociaux auxquels il est relié. La " mise en agenda politique " des problèmes environnementaux (Gilbert, 2003), la façon dont ils sont identifiés, mesu- 
rés et mis en débat (Callon et al, 2001), ou encore les politiques et initiatives qu'ils motivent ainsi que les conséquences qu'ils entrainent pour les sociétés et leur espace constituent, par exemple, des objets d'étude pertinents pour les sciences sociales qui se penchent sur l'environnement.

Comme science des relations, la géographie s'intéresse de façon privilégiée aux interactions multiples -tant matérielles qu'immatérielles- entre les sociétés et leur environnement. Ainsi, la question environnementale permet d'observer le renouvellement de la pensée aménagiste (Berdoulay et Soubeyran, 2000) tout comme elle fait l'objet d'analyses plus opérationnelles sur les modalités d'application des politiques locales d'adaptation (Bertrand et Larrue, 2007), notamment du fait du Grenelle Environnement à l'origine des lois dites Grenelle 1 et 2 adoptées en 2009 et 2010. Ce dispositif légal constitue le cadre national d'opérationnalisation de directives européennes adoptées en 2007-2008 concernant l'énergie et le climat. Pour autant, l'Union Européenne n'est pas nécessairement l'aiguillon principal des initiatives nationales (Biesbroek et al, 2010). La France a par exemple adopté sa stratégie nationale d'adaptation en 2007 et installé l'Observatoire National sur les Effets du Réchauffement Climatique (ONERC) en 2001. C'est le troisième rapport du GIEC qui, en 2001, mobilise la notion d'adaptation parmi les catégories d'analyse des défis environnementaux afin de dépasser les limites d'une approche globale et physique de la problématique du changement climatique, jusque là centrée sur l'atténuation des gaz à effet de serre. En la définissant comme un ajustement des systèmes humains et naturels à un environnement changeant, le GIEC renoue avec un usage de la notion d'adaptation comme catégorie classique de l'étude du milieu géographique et des interactions entre sociétés et environnement (Berdoulay, 2009). Au fil des dix dernières années, l'adaptation s'impose autant parmi les institutions internationales qu'au sein de la production scientifique ou dans les cadres légaux et règlementaires nationaux. Catégorie pivot, relevant à la fois de l'analyse et de l'action, l'adaptation au changement climatique se singularise notamment par deux aspects : d'une part le passage du "lutter contre " (propre à l'atténuation) au " vivre avec ", et d'autre part l'accent mis sur le local, à travers la déclinaison de stratégies d'action et la mise en place de dispositifs sociaux, techniques et institutionnels à grande échelle. L'accent est mis sur les territoires, sur l'appropriation des politiques, sur la mise en place de stratégies locales d'adaptation. Plus précisément, les lois Grenelle enjoignent les collectivités territoriales de plus de 50000 habitants à adopter des documents règlementaires et stratégies locales d'adaptation au changement climatique avant la fin de l'année 2012. On trouve parmi ces outils les Plans Climat Energie Territorial (PCET), censés intégrer aux projets territoriaux des enjeux liés à l'énergie et au changement climatique sous la forme de stratégies, de mesures concrètes ou de plans d'action. Sur le terrain, les collectivités territoriales sont saisies par cet impératif légal qui consiste à décliner les PCET sur leur territoire.

Ainsi, le contexte contemporain des rapports au milieu et de leur gestion peut être caractérisé par la redéfinition des acteurs jusque là légitimes à justifier l'action, par une réflexion sur la solidarité et les enjeux collectifs que recouvrent les défis environne- 
mentaux, et par le fait que d'importants secteurs de la recherche s'emploient à dépasser les modèles descendants d'action publique et à penser des dispositifs plus collaboratifs et inclusifs. Dans ce contexte, et à partir de l'application des politiques d'adaptation sur les territoires locaux aquitains, on se demande dans quelle mesure la question environnementale constitue (ou peut constituer) l'occasion d'un renouvellement de l'action publique et de la planification. Quels sont les principaux enjeux posés sur le terrain par les impératifs légaux d'adaptation? Quelles modalités d'application - et leurs limites - constate-t-on sur le terrain ? La mise en place des stratégies locales d'adaptation en Aquitaine est-elle l'opportunité d'un retour sur la gestion territoriale, la démocratie locale et les rapports au milieu, ou est-on face à des formes de continuité ou de reproduction qui seraient le fait d'initiatives superficielles et ponctuelles, relativement étrangères aux contextes territoriaux? En définitive, dans quelle mesure la question de l'adaptation introduit des éléments originaux en matière d'action publique locale et de gestion territoriale?

Les éléments de réponse apportés à ces problématiques reposent sur une partie des résultats et de l'interprétation de la première phase d'analyse du programme CESAR (Changement Environnemental et Stratégies d'Adaptation en Région - http://set.univpau.fr/live/cesar). Ce programme vise d'une part à évaluer l'opérationnalisation de l'initiative nationale d'adaptation introduite par les lois Grenelle de façon descendante, sur les territoires d'Aquitaine, et d'autre part à analyser les initiatives et innovations ascendantes en lien avec l'adaptation au changement climatique, et propres aux acteurs locaux, élus, professionnels, techniciens, mais aussi citoyens, en complément de, en parallèle à ou en concurrence avec l'impératif légal des lois Grenelle. Si d'autres territoires locaux, pionniers, ont fait l'objet d'études fournies (comme c'est le cas pour la Communauté urbaine du Grand Lyon, Bertrand et Rocher, 2011), les territoires aquitains méritent une attention particulière. En effet, les pouvoirs publics régionaux s'avèrent réactifs dans ce domaine (pionniers dans l'élaboration d'un plan climat régional en 2007), conscients des enjeux que présente le changement climatique pour des activités phares en région, comme le tourisme (d'hiver ou balnéaire), la viticulture ou la monoculture de pin dans le massif des Landes, et au fait des défis posés par une forte littoralisation du peuplement et des activités. Outre l'ensemble des terrains réalisés à travers la région, nombre d'exemples et de données collectées sont tirés de l'espace pyrénéen. La tendance à l'assèchement s'y avère particulièrement sensible, en particulier pour l'activité agropastorale, et des initiatives transfrontalières intéressantes -mais encore au stade de l'ébauche- dans le domaine de l'adaptation au changement climatique pourraient faire l'objet d'analyses ultérieures dans un contexte de collaboration transnationale (on pense notamment à l'Observatoire Pyrénéen du Changement Climatique -http://www. opcc-ctp.org/- qui promeut des initiatives de coopération multirégionale et tri nationale autour d'enjeux environnementaux).

L'analyse proposée présente des enseignements d'ordre opérationnel et épistémologique. D'une part, elle concerne les recompositions de l'action publique locale à la 
lumière des modalités d'application et des difficultés d'appropriation des stratégies d'adaptation observées sur le terrain aquitain. D'autre part, elle interroge la capacité des sciences sociales à se saisir de la problématique de l'adaptation (et plus largement de la question environnementale) comme d'un fait social total, i.e. de leur capacité à interpréter les stratégies locales d'adaptation à partir des spécificités socio territoriales auxquelles ces stratégies sont reliées, et non exclusivement sur la base de modalités ou de configurations tirées d'une rationalité étrangère aux logiques territoriales.

Dans la perspective de ce double enjeu, la réflexion proposée se présente en deux temps. Le premier temps du diagnostic repose sur la constitution et l'analyse au printemps 2012 d'un corpus de documents liés à l'adaptation au changement climatique et tirés des sites Internet des collectivités territoriales d'Aquitaine, sur l'observation, durant l'automne 2011 et l'hiver 2012, d'initiatives mises en place sur le terrain ou encore de leur réception parmi les acteurs locaux, ainsi que sur des entretiens individuels avec des techniciens de collectivités concernées par les lois Grenelle, entretiens pratiqués tout au long de l'année 2012. Le deuxième temps de l'interprétation des enjeux épistémologiques et opérationnels liés à la mise en place des stratégies d'adaptation s'inscrit dans les postures du débat académique sur l'action publique et les approches de l'environnement par les sciences sociales pour suggérer des questions de recherche plus pertinentes au regard des premiers enseignements de terrain. Le cas échéant, il est aussi question de suggérer des domaines d'intervention privilégiés pour une opérationnalisation de l'adaptation plus juste, mieux appropriée, et plus en phase avec les caractéristiques territoriales.

\section{Regards sur l'adaptation dans les politiques des collectivités locales}

\subsection{Le poids des cadres normatifs et règlementaires}

L'élaboration ou la mise en place des stratégies d'adaptation au changement climatique rencontrées sur les terrains aquitains s'inscrivent dans le cadre des lois Grenelle. À ce titre, elles correspondent à un même cadre, utilisent les mêmes termes, mobilisent les mêmes outils et reproduisent des formats très proches. On pense notamment aux outils obligatoires comme les cadres identiques des PCET ou les diagnostics carbones qui consistent en une évaluation des émissions de $\mathrm{CO} 2$ d'un territoire, ou d'une activité. Mais ces dispositifs se déclinent sous la forme de "fiches action ", d'exercices de concertation, d'un souci explicite d'exemplarité, d'une obligation de sensibilisation et de formation interne aux collectivités, puis d'une même obligation à l'externe, auprès des citoyens. On retrouve ces modalités d'application presque clonées, sur les différents territoires locaux observés. Il en va de même pour la valorisation de "l'éco citoyenneté " et des "bons " comportements ou la mise en avant de l'économie verte, le tout sur fond d'un constat identique d'urgence de l'action (Salvestroni, 2012). La mul- 
tiplication de pratiques et d'outils similaires compte notamment sur le concours des services de l'État, comme l'ADEME (agence de l'environnement et de la maitrise de l'énergie, établissement public) ou la DREAL (direction régionale de l'environnement, de l'aménagement et du logement, échelon régional du ministère de l'écologie, du développement durable et de l'énergie) qui assurent un soutien technique ou méthodologique ponctuel sur lequel les collectivités territoriales s'appuient. Par un document en ligne daté d'août 2010, le Conseil Général du Lot-et-Garonne annonce par exemple décliner les objectifs du plan climat Aquitain avec les soutiens techniques et financiers de la Région Aquitaine, de la DREAL, de l'ADEME et de l'Europe ${ }^{1}$.

Certes, la loi est la même pour tous sur le territoire de la République. Pour autant, l'impératif d'adoption des stratégies d'adaptation ne fait pas sens également partout, sur les différents territoires locaux, caractérisés par des acteurs et des contextes spécifiques. Si la loi est la même, la territorialisation des politiques et l'opérationnalisation des dispositifs d'adaptation à l'échelle locale semblent se plier difficilement à la reproduction presque aveugle d'un cadre d'application descendant. La reprise peu nuancée d'un cadre indifférencié interroge en matière d'appropriation, d'adhésion et d'efficacité de la mise en place des stratégies d'adaptation sur les territoires. Sur le terrain, on se trouve face à une double caisse de résonnance. D'une part, on assiste à la reproduction des dispositifs règlementaires, via le concours des services de l'État le cas échéant. D'autre part, lorsqu'on se penche sur les pratiques développées et les actions menées sur le terrain, on retrouve les principes caractéristiques du développement durable, très installés sur les territoires, dans les consciences, et parmi les références pour l'action des collectivités territoriales. Les notions mobilisées et qui font sens sur le terrain sont plus liées au développement durable qu'à l'adaptation. Cet autre effet de résonnance, de réplication des principes du développement durable, pourrait faciliter l'ancrage d'autres préoccupations environnementales collectives, autour de l'adaptation et du changement climatique.

Pour autant, dans l'immédiat tout du moins, on ne relève pas d'imaginaire de l'adaptation sur les territoires. Les préoccupations locales liées au changement climatique existent, mais rencontrent mal les priorités telles que formulées en termes d'atténuation, d'énergie et de climat par les outils du cadre légal du Grenelle. La tension entre la réplication des dispositifs règlementaires d'une part, et d'autre part la déclinaison locale et l'appropriation difficiles des stratégies d'adaptation peut trouver une partie de son explication dans cette non-correspondance entre les objectifs règlementaires de l'adaptation et ce que l'enjeu de l'adaptation au changement climatique recouvre sur les territoires.

1. Consultation du site du Conseil Général du Lot-et-Garonne le 12 juin 2012. http://www.cg47.fr/ 


\subsection{L'adaptation: un principe transversal ou traversé par d'autres problématiques?}

Dans nombre de collectivités locales, et des dires des techniciens enquêtés, le développement durable est posé comme un préalable aux politiques territoriales, et on observe une tendance similaire avec l'adaptation au changement climatique. Du reste, les Plans Climat Energie Territoriaux sont considérés comme des projets territoriaux constitués à la lumière des enjeux climatiques et énergétiques identifiés sur les territoires. Le mainstreaming de l'adaptation au changement climatique est le principe qui consiste à faire passer par le filtre des enjeux et des priorités que pose l'adaptation l'ensemble des politiques, des projets, des initiatives du territoire. Or sur le terrain, il s'avère que bien plus qu'un point de départ à partir duquel penser le projet territorial, il est tout aussi possible de penser l'impératif d'adaptation au changement climatique comme traversé par une série d'autres problématiques. Ainsi, plus que ses modalités d'application ou les formes de ses stratégies, c'est la pluralité de ce que recouvre l'adaptation pour les différents acteurs locaux qui fait question. Sous couvert d'urgence et d'unanimité, l'adaptation véhicule alors, en les "neutralisant ", en les dépolitisant, des enjeux locaux qui font l'objet de controverse et qui semblent constituer un obstacle sérieux à l'appropriation des stratégies d'adaptation. Plusieurs observations viennent à l'appui de cette lecture.

De façon formelle, il peut sembler paradoxal de présenter la question environnementale, et plus particulièrement d'afficher la problématique de l'adaptation au changement climatique comme transversale aux politiques locales d'une part, et de la décliner isolément dans chacun des secteurs d'activités et d'intervention des collectivités territoriales d'autre part, comme il apparaît dans nombre de documents mis en ligne par les collectivités.

En second lieu, la capacité des politiques environnementales locales à reconnaître et prendre en compte des problématiques spécifiques aux territoires semble faire une différence importante. Ne pas considérer ou même dissimuler des particularités locales pourtant affectées par les stratégies d'adaptation s'avère être un obstacle notable pour que nombre d'acteurs locaux y adhèrent. Or l'impératif règlementaire d'adaptation au changement climatique ne va pas de soi au regard de la pluralité des rationalités et des priorités sur les territoires. La notion d'adaptation, comme catégorie d'analyse, mais aussi d'action, recouvre de multiples dimensions qu'il est important de ne pas négliger. Ainsi, la dimension conceptuelle de l'adaptation reste floue sur le terrain. Elle est peu partagée parmi les acteurs de terrains. Les techniciens se disent assez dépourvus de compétences et d'outils dans le domaine, alors que sa mise en application se rapproche souvent du cadre de références du développement durable. Une dimension politicienne se joue aussi, liée à des enjeux d'opinions ou d'échéances électorales, notamment à travers l'affichage d'une participation qui souvent se limite à une consultation, voire à un simple " porter à connaissance ". Certains techniciens et élus locaux et régionaux enquêtés ont mis en avant l'inadéquation entre agendas des politiques environnementales 
et agendas électoraux. À cette inadéquation somme toute classique, mais qu'il est impossible d'ignorer, s'ajoute une dimension géopolitique locale au cœur de logiques de décentralisation et de réorganisation des pouvoirs locaux à travers la question environnementale. Par exemple la région, qui n'a pas de mandat particulier dans la mise en place des stratégies locales d'adaptation, affiche une posture proactive qui peut être tout autant la marque de son intérêt pour la thématique que le souci d'occuper une position politique significative entre l'État et le local. On retrouve notamment les processus déjà documentés des transformations de l'exercice du pouvoir central, par le passage de l'intervention à la régulation à travers la multiplication d'impératifs légaux délégués aux échelons locaux (While et al., 2010). En outre, les rapports de pouvoir qui se jouent entre l'État et la région ou la région et les collectivités territoriales se retrouvent aussi entre les collectivités territoriales et les communes qui les composent. C'est par exemple le cas de municipalités de la Côte Basque récalcitrantes au transfert de leurs prérogatives sur le foncier et l'usage du sol aux intercommunalités dont elles font partie. Bien qu'il s'agisse d'un instrument fondamental pour penser un projet territorial intercommunal - en ligne avec des objectifs d'adaptation, le contrôle du sol recouvre également des intérêts capitaux pour les communes dans un contexte de pression extrême sur le foncier. De là, la dimension économique de l'adaptation devient évidente. La perspective de la modernisation écologique offre des opportunités pour les territoires, pour le développement de nouveaux métiers (comme les bureaux d'étude investis dans le conseil aux collectivités dans l'environnement, ou dans la réalisation des diagnostics et bilans carbone pour lesquels les collectivités ne présentent pas toujours de compétences suffisantes en interne). En outre, à l'échelle de la région Aquitaine, les pouvoirs publics comptent les marchés volontaires de carbone dans leur éventail de stratégies d'adaptation, notamment pour donner une nouvelle dimension à la reforestation des Landes suite à la tempête Klaus, de 2009. Cette option stratégique se traduit par un investissement public d'un demi-million d'euros à travers l'association Aquitaine Carbone, levier de cette initiative. D'autres enjeux locaux encore se jouent à travers l'adaptation au changement climatique, et notamment autour d'une dimension politique liée à l'exercice de la démocratie locale, au recours à la concertation, à la consultation publique, et autour de l'enjeu de la parole et de l'initiative laissée aux administrés et aux citoyens. Les différentes collectivités enquêtées ne présentent pas les mêmes procédures de consultation et n'offre pas le même périmètre de participation, d'intervention ou de contrôle à leurs administrés. Une dimension de justice, enfin, ressort de certains échanges tenus à l'occasion de réunions publiques à propos des leviers adéquats pour l'application de certaines stratégies d'adaptation. Le domaine particulier de l'incitation fiscale ou de l'obligation légale a notamment soulevé ces questions: comment ne pas pénaliser les populations les plus précaires (par une obligation d'équipement, d'investissement, ou par la taxation de certaines pratiques) ? Comment lutter contre la distribution socialement différenciée des efforts d'adaptation et des implications des politiques territoriales?

À la lumière de cette énumération non exhaustive tirée des observations et des entretiens de terrain, il apparaît que bien d'autres problématiques que les aspects stric- 
tement environnementaux, climatiques ou opérationnels traversent les stratégies locales d'adaptation, au-delà de l'impératif légal qui les marque. La compréhension de la notion, la façon de poser les grands enjeux à traiter, ou encore les types de réponses envisagées sont autant d'éléments préliminaires, a priori, qui favorisent ou entravent la reconnaissance et la prise en compte des spécificités des territoires concernés. Plus que sur le "comment faire" (la question opérationnelle et technique qui se penche sur le procédé adéquat de mise en place des stratégies), l'attention se porte sur l'importance de la diversité des rationalités ainsi que des territoires à l'aune desquels l'impératif d'adaptation au changement climatique doit nécessairement être pensé. C'est à un renversement de la problématisation de l'adaptation au changement climatique que nous amènent les premiers résultats de ces terrains aquitains.

\subsection{De l'analyse à l'action, paradoxes et enjeux de la notion d'adaptation}

Le paradoxe à pointer est celui du relatif succès de l'adaptation dans le débat académique, pour l'action de nombre d'institutions internationales ou pour le financement d'initiatives, face à quoi se dresse l'absence d'imaginaire et de références à l'adaptation sur le terrain et pour l'action, parmi les acteurs locaux. L'adaptation s'avère ramenée aux principes du développement durable dont on dit, parmi les élus et techniciens enquêtés des collectivités aquitaines, qu'ils sont désormais bien ancrés dans les pratiques et comme cadres de l'action. La référence au développement durable est plus familière, plus immédiate, plus appropriée localement. Il en va autrement de l'adaptation, malgré la forte promotion de la notion à petite échelle. De façon particulièrement représentative d'un sentiment partagé sur le terrain, un technicien d'une structure locale accompagnant l'activité agricole en Pyrénées a formulé l'interrogation suivante : "où est-ce que 'l'adaptation' [puisque l'enquêteur employait le terme] et plus largement où est-ce que les politiques environnementales sont pensées? ". L'impératif d'adaptation qui arrive sur le terrain semble alors tomber du ciel. Cet impératif amènerait à sortir du cadre du développement durable, certes difficile à opérationnaliser, mais approprié après plusieurs années de sédimentation, et à devoir élaborer et mettre en place des stratégies d'adaptation dont on saisit mal la spécificité. À travers cette interrogation, les acteurs locaux (et pas seulement les techniciens des collectivités territoriales) se sentent étrangers aux arènes où sont discutés et élaborés les cadres des politiques d'adaptation qui s'imposent sur les territoires en prenant fort peu en compte les réalités et les problématiques locales. Or, le paradoxe entre un succès (même relatif, dans certaines sphères) et une très faible appropriation sur le terrain retourne de la nécessité de situer les cadres et stratégies d'adaptation au sein des différents territoires. C'est là un enjeu majeur qui semble ressortir des premières expériences de mise en place des stratégies d'adaptation promues par le Grenelle environnement.

Cet enjeu de territorialisation est d'autant plus fondamental que les politiques locales d'adaptation interviennent dans des contextes où les opinions semblent favorablement 
travaillées par les principes du développement durable. En effet, la mise en débat ou l'existence d'arènes de discussion autour des stratégies locales d'adaptation permettrait d'attirer l'attention sur deux points fondamentaux (la pluralité des rationalités et la solidarité ou l'articulation multi scalaire) qui méritent d'être mieux connus de façon à œuvrer pour une meilleure appropriation des stratégies d'adaptation sur les territoires :

- D’une part, il serait possible de reconnaître la pluralité des rationalités, des compréhensions de la notion d'adaptation, et plus largement du défi environnemental et climatique qui s'expriment sur le terrain, et qui font sens au regard des spécificités locales. La question de l'adaptation non pas " comment ", mais "pour qui " mérite d'être posée au risque d'ignorer (sinon de mépriser, comme c'est parfois ressenti) nombre de réalités et de logiques locales. Il arrive que les acteurs sur le terrain se détournent des objectifs environnementaux affichés, pas nécessairement par manque de conviction, mais en définitive du fait de modalités d'application qui ignorent les territoires (pour éviter des contraintes règlementaires, contourner des priorités de financement, ou en réaction à une hiérarchie de problématiques jugées iniques).

- D'autre part, la reconnaissance d'enjeux et de priorités multiples sur les territoires amènerait à cadrer différemment les politiques d'adaptation souvent perçues comme l'application froide d'un agenda règlementaire aveugle aux logiques locales. Ce regard par le territoire n'est pas pour autant synonyme du travers inverse, à savoir l'enfermement dans un localisme étranger à des principes de solidarité ou ignorant les enjeux posés à d'autres échelles (un des moteurs des principes d'adaptation : " agir local, penser global "). De fait, l'expérience rapportée par un agronome de la chambre basque d'agriculture (EHLG) ${ }^{2}$ montre que la quasi-totalité des exploitants de l'espace concerné par EHLG et interrogés à propos de la pollution des eaux de rivière du fait de pratiques à risque est tout à fait prête à considérer des intérêts qui ne leur sont pas immédiats ni particuliers, et à remettre en cause leurs pratiques.

Au regard du paradoxe entre le succès d'une part et la défiance d'autre part, un enjeu majeur des politiques locales d'adaptation au changement climatique repose sur la capacité à restituer la pluralité des territoires, des initiatives, de l'action, des mobilisations, des différentes raisons d'agir et finalement des stratégies à mettre en place face à la singularité de la problématique du changement climatique. L'interprétation du diagnostic de terrain qui est présentée dans la partie suivante s'inscrit dans une perspective plus ouverte et plus opérationnelle, tel que le programme CESAR en a la vocation. Il s'agit notamment de dépasser le paradoxe et la tension identifiés, de penser une convergence entre le pluriel des territoires d'une part et le singulier d'une probléma-

2. Entretien réalisé le 12 novembre 2012. 
tique climatique d'autre part, en sachant que cette problématique présente des difficultés historiques à être déclinée localement (Demeritt, 2001), et qu'elle se pose dans le contexte de pratiques héritées d'action publique descendante que l'on cherche à actualiser (Berdoulay et Soubeyran, 2012).

\section{L'appropriation de l'adaptation sur les territoires aquitains: principaux obstacles et enjeux}

\subsection{Les contours classiques du débat environnemental à dépasser sur le terrain}

Dans le domaine des controverses environnementales, et concernant notamment les choix à opérer et les initiatives à privilégier face au changement climatique, le débat et les options qui se présentent sont souvent réduits à une dialectique entre défi technique et défi sociétal (Blanc et Lolive, 2007). L'alternative à laquelle est souvent réduit l'enjeu environnemental se caractérise moins par des partis pris idéologiques que par une très faible remise en question de l'action publique et des modalités de planification locale. En effet, le défi technique mise sur l'intensification d'un rapport de domination, sinon de maîtrise, des sociétés sur leur environnement en faisant le pari de la technologie et de l'innovation comme issue principale des problèmes environnementaux. Cette posture optimiste quant à la capacité des sociétés à innover et à dépasser ces défis par la technique est tout autant incarnée par le principe espérance (Bloch, 1976), porteuse d'un sens de l'histoire que les sociétés accomplissent, que par les principes libéraux (Ferry, 1992) marqueurs de la foi dans les capacités des individus à se réinventer. L'une et l'autre de ces positions sont en ligne avec une posture moderne caractérisée par l'affirmation de la séparation entre sociétés et milieu naturel, et par le récit d'une nécessité de l'histoire, d'un progrès à accomplir. Face au défi technique, le défi social oppose les responsabilités du fonctionnement des sociétés, des modes de déplacement et de consommation, qui seraient à l'origine des défis environnementaux auxquels il faut faire face. Apparemment plus encline à concevoir des interactions complexes entre sociétés et milieu, la posture du défi social reste très axée sur l'organisation et le fonctionnement des sociétés (plus que sur les interactions avec le milieu), et mobilise pour son argumentation les mêmes registres d'expertises et de discours savants auxquels cette posture oppose pourtant une entrée plus sociale et humaine. C'est par exemple le cas de l'élaboration du DOCOB (Document d'Objectifs, aux fonctions de diagnostic et d'orientation) dans la vallée de Baigorry, préalable à la mise en place d'un dispositif Natura 2000 destiné à maintenir la diversité biologique remarquable dans une perspective de développement durable. Deux camps s'opposent pour défendre, les uns, un périmètre réduit, les autres, un périmètre bien plus étendu pour déployer le dispositif Natura 2000. La querelle ne repose pas sur la pluralité des dimensions (sociales et humaines) que peut recouvrir la controverse environnementale, comme on a pu l'illustrer précédemment (alors même que le DOCOB comporte un volet socioéconomique). 
Elle se réduit plutôt à une seule bataille d'expertises et contre-expertises, et notamment de confrontations de méthodes destinées à dresser le diagnostic agropastoral (influençant par conséquent le contenu "d'expertise "sur la base de quoi le périmètre de conservation est argumenté) $)^{3}$. Il en va de la controverse autour du dispositif Natura 2000 de Baigorry comme de la mise en place des stratégies d'adaptation au changement climatique sur le terrain. Les contours du débat environnemental, à travers une réduction aux défis technique ou social, s'inscrivent dans les lignes d'une action publique descendante, et donnent la priorité aux savoirs savant ou expert. Certes, un plus grand nombre d'acteurs peut aujourd'hui se saisir d'instruments significatifs et adopter des postures déterminantes pour l'action (légitimité, autorité, expertise, jeux d'opinion), mais ni le modèle descendant d'action et de décision ni les registres dominant de l'argumentation ou de la conceptualisation ne sont remis en cause.

De fait, sur les territoires aquitains, les aspects techniques sont beaucoup développés au titre des stratégies d'adaptation. Les fiches action ou les objectifs ponctuels (comme la viabilisation de pistes cyclables) sont plus faciles à mettre en œeuvre et plus visibles politiquement. Pour autant, l'essentiel des collectivités territoriales ne manque pas de faire figurer au moins dans leurs documents en ligne et dans leur communication la nécessité pour les acteurs locaux de changer de comportements, de modes de vie, de modes de production ou encore de modes de déplacement. Les deux postures, technique et sociale, coexistent. Dans l'un comme dans l'autre cas, la perspective est très tournée vers l'action, et descendante. En effet, pour beaucoup, les pouvoirs publics - et ici les collectivités territoriales - représentent l'intérêt général institué, et par conséquent l'arène la plus adéquate pour répondre aux défis environnementaux et mettre en place des stratégies d'adaptation sur le terrain. Cette idée de l'action publique descendante dans le domaine de l'adaptation est reprise et véhiculée avec plus ou moins d'évidence en fonction des collectivités. Ainsi, on retrouve très clairement cette idée dans le PCET de la communauté d'agglomération du bassin d'Arcachon :

La moitié des émissions de gaz à effet de serre est produite par les habitants dans leurs gestes quotidiens. Il est donc essentiel que les pouvoirs publics interviennent au travers de politiques intégrant la question du changement climatique, afin d'impliquer tous les individus et changer les comportements. En effet, ce sont les collectivités qui possèdent la plus grande capacité d'action (technique, administrative) envers les habitants. Elles jouent un rôle majeur au travers des leurs compétences directes, mais aussi en termes d'exemplarité, d'incitation, d'animation et de communication (COBAS, $2012: 7-8$ ).

En revanche, dans le rapport de 2011 sur le développement durable de la communauté d'agglomération Côte Basque - Adour, on ne part pas d'une collectivité territo-

3. Entretien réalisé le 7 novembre 2012 avec un en charge de l'élaboration d'une partie du DOCOB. 
riale fondée à orchestrer le collectif. On lit plutôt qu'une finalité des politiques publiques et de l'action de la communauté d'agglomération consiste à tendre vers :

[La] satisfaction des besoins essentiels [et] la qualité de vie des habitants [...pour des] habitants de l'agglomération soucieux de la préservation de leur cadre de vie qu'ils jugent de qualité. [La communauté d'agglomération fonctionne] dans une approche plus globale de répondre aux besoins des habitants (Agglomération Côte Basque Adour, 2012 : 19).

L'idée semble être plutôt de partir des besoins des habitants et du terrain pour orienter les politiques publiques. Certes, reste à savoir comment les pouvoirs publics peuvent se faire écho de quels besoins, et des besoins de quels secteurs de la population. Les réalités que recouvre l'idée de participation, depuis la période de concertation pour l'élaboration du Grenelle Environnement (Boy, 2010) jusqu'à la mise en place des stratégies locales d'adaptation, méritent un travail de terrain minutieux et des données de première main. Pour autant il semble qu'entre la COBAS et la CA Côtes Basque -Adour un même débat environnemental (autour de questions larges de durabilité, d'adaptation et de changement climatique) prend des contours différents au regard des rapports qui se dessinent entre pouvoirs publics, populations locales et exercice de la démocratie.

\subsection{Les multiples limites d'une action publique descendante}

Le modèle descendant de l'action publique, en l'occurrence dans le domaine de l'adaptation au changement climatique, est mis en question à partir des territoires de trois façons principales :

Sur le terrain, on constate l'émergence d'autres acteurs légitimes que les acteurs centraux du pouvoir constitué ou que les acteurs porteurs du savoir expert dans l'élaboration et la prise de décision. Le mouvement de la décentralisation et la consolidation des gouvernements locaux accompagnent la consolidation de la société civile, de la population organisée -sous forme d'associations, de groupes de pression, de comités de participation- dont la voix fait désormais partie d'une gouvernance largement recomposée (Theys, 2003). De fait, d'autres agendas se jouent de façon plus explicite sur le terrain. Cette nouvelle géopolitique locale vient brouiller les pratiques descendantes des pouvoirs publics dans la gestion environnementale. Mais au-delà des divers intérêts qui se manifestent, on constate aussi une plus grande visibilité de différentes rationalités. Les acteurs émergents manifestent des besoins, des conceptions, des priorités qui certes ne sont pas nécessairement déterminants, mais qui comptent dans le paysage de l'action publique. La prise en compte de ces voix (selon des modalités qui sont à inventer) pourrait même constituer une condition d'appropriation et de réussite des politiques locales. 
Dans un autre registre, la conscience des enjeux environnementaux semble avoir gagné du terrain localement. Ce nouveau terreau de réception pourrait s'avérer très favorable à la mise en place des politiques d'adaptation au changement climatique. Pour autant, comme illustrée plus haut, la catégorie de l'environnement n'est pas nue et pure. Elle s'inscrit dans un contexte, associe divers enjeux et des acteurs aux conceptions et priorités différentes. À travers l'impératif de l'adaptation au changement climatique, d'autres enjeux se nouent, propres aux territoires, qui remettent en question l'urgence et l'exclusivité à l'endroit d'un seul défi environnemental souvent mis en avant dans les discours dominants. Les acteurs locaux font preuve d'une certaine réflexivité, mais les recompositions de la modernité (Beck, 2003 ; Innerarity, 2008) sont aussi marquées par le doute quant à la capacité d'une action publique descendante à promouvoir des stratégies d'adaptation qui rencontrent les spécificités des territoires. Ainsi, la défiance parfois rencontrée sur le terrain à l'endroit des politiques d'adaptation pourrait s'expliquer plus sûrement par la crise de confiance dans l'application de solutions descendantes peu attentives aux territoires que par le manque d'intérêt que les acteurs locaux portent aux défis posés par le changement climatique.

Enfin, et pour preuve d'une conscience environnementale très pragmatique sur le terrain, les observations et les entretiens révèlent de nombreuses initiatives d'acteurs locaux, de professionnels ou de simples citoyens qui ne comptent pas nécessairement avec l'impulsion ni le concours des pouvoirs publics. Les indices d'un renouvellement possible de l'aménagement et de la mise en place des politiques publiques par les territoires peuvent se dessiner à travers l'adaptation et l'enjeu environnemental. Parmi ces expériences propres aux terrains, on trouve par exemple la remise en service des canaux d'irrigation pour certaines prairies à foin des vallées pyrénéennes. Cette actualisation d'un usage passé peut être considérée pour partie au moins comme une réponse à la diminution sensible des précipitations afin d'assurer plusieurs fauches (Evrard, 2009). Éleveurs et élus locaux, notamment dans la vallée d'Aspe, ont signalé la reprise de l'entretien des pachères. Les pachères sont des petits canaux creusés et dragués avec une petite pioche par les professionnels de l'élevage lorsqu'ils parcourent la montagne afin de drainer les terrains en pente ou orienter l'écoulement de surface. Pour beaucoup, la négligence de ces pachères a contribué à de nombreuses déconvenues (sols engorgés, arrachement, flux torrentiels) qu'il conviendrait de corriger par un meilleur " ménagement " du milieu. Dernier exemple, la mécanisation de l'activité sylvicole depuis un demi-siècle a mené à l'abandon de certaines parcelles trop accidentées dont l'exploitation du bois à l'aide de machines était rendue impossible. Dans une logique de réappropriation de la montagne et de lutte contre la fermeture des paysages (certes aussi en fonction des opportunités économiques, fiscales ou marchandes), on constate la remise en service de câbles destinés à relier les coupes des parcelles accidentées à un point accessible aux camions et au réseau routier. Toutes ces expériences sont autant de réponses à un environnement qui change, nécessairement, et pas du seul fait des dynamiques physiques du milieu naturel. Ces stratégies d'adaptation qui émergent 
pour beaucoup du terrain ne sont pas un retour vers des pratiques anciennes, vers le "bon temps " ni vers l'usage traditionnel. Elles semblent être plutôt une actualisation performative de pratiques héritées, certainement idéalisées, et qui mériteraient des recherches spécifiques. Ainsi, bien plus que le fait des stratégies locales d'adaptation du Grenelle Environnement, la gestion du milieu en "bonne intelligence " semble être, pour certains interlocuteurs, l'apanage des forales béarnais (gestion traditionnelle et partagée des ressources de la vallée, vieille de "plusieurs siècles "4), de la gestion des communs dans les hautes vallées (aujourd'hui encore, dans la vallée d'Aspe, l'usage des estives de la commune de Borce fait par exemple l'objet d'une gestion collective ${ }^{5}$ ), ou encore de la tradition de la démocratie basque exercée sur le "perron de l'église ", entre voisins ${ }^{6}$. Ces récits travestissent certainement la nature originale de ces pratiques héritées pour les transposer à la période contemporaine. Mais ils sont la marque d'une intelligence locale, de rapports au milieu, et d'une conscience aiguë d'un environnement qui change, et auxquels l'action publique pourrait gagner à s'articuler.

En définitive, plus que le contenu et les objectifs des politiques d'adaptation, ce sont les formes, les conditions, la réception de ces dispositifs et initiatives sur le terrain qui constituent les freins les plus importants à leur appropriation. Fort d'un recul de quelques années, plusieurs interlocuteurs enquêtés, tant parmi les collectivités territoriales que sur le terrain, ont signalé l'importance accordée par chacun à la problématique environnementale, et plus encore au cours des 5 dernières années. Ainsi, si " les choses expliquées et comprises sont mieux acceptées " 7 , il ne faut pas négliger les façons dont chacun fait et vit son territoire, reconnaît et comprend les enjeux environnementaux. Il ne s'agit pas seulement de convaincre, d'éduquer ou de sensibiliser. Il s'agit surtout de reconnaître des rationalités plurielles (ce qui n'empêche pas de porter un regard critique et différentié à leur endroit) et de trouver les vecteurs adéquats d'échanges, puis d'appropriation et d'application des dispositifs et stratégies d'adaptation sur le terrain. Ce premier pas de l'appropriation, la reconnaissance, pourrait être l'opportunité de construire collectivement, à partir des multiples ressources territoriales, une réponse appropriée et significative pour tous, et non pour chacun. Sur la base de cette lecture, on cherche à adopter des approches de sciences sociales attentives aux dimensions territoriales et collectives de la question environnementale afin de produire une connaissance significative pour l'action. Il s'agit d'identifier les conditions nécessaires à l'élaboration de dispositifs collaboratifs, propres aux territoires concernés.

\footnotetext{
4. Entretien réalisé le 8 novembre 2012.

5. Entretien réalisé le 7 novembre 2012.

6. Entretien réalisé le 8 novembre 2012.

7. Entretien réalisé le 7 novembre 2012 auprès d'un élu de la vallée d'Aspe.
} 


\section{Un premier bilan pour l'adaptation : défis de l'appropriation et double enjeu épistémologique et politique}

Des techniciens de collectivités territoriales constatent qu'il est désormais très hasardeux -voire impossible-d'envisager la mise en place d'initiatives ou de politiques locales étrangères aux préoccupations environnementales devenues transversales à l'action publique sur le terrain. Dans ce cadre, l'introduction progressive de la notion peu connue d'adaptation pose de nombreuses questions d'ordre opérationnel. Mais qu'il s'agisse de pression de l'opinion, de cadres règlementaires ou de conditionnement des financements, les politiques locales sont nécessairement articulées aux préoccupations environnementales. En revanche, afficher une considération certaine pour les préoccupations environnementales peut tout aussi bien mener à différents types d'action, dont les motivations sont parfois bien éloignées des problématiques environnementales. Le régime de justification peut évoluer, mesures et politiques locales font preuve d'un fort degré de continuité le cas échéant ${ }^{8}$.

Le principal défi que présente la mise en place de stratégies d'adaptation au changement climatique sur le terrain concerne les multiples ressorts, sociaux et humains, dont elles relèvent. Ces ressorts s'avèrent peu pris en compte par des politiques descendantes qui tendent facilement à négliger, à ignorer ou à écraser les rationalités plurielles sur le terrain et la complexité des territoires. Le contexte contemporain est pourtant caractérisé par une reconnaissance partagée du défi environnemental parmi les acteurs locaux (tant sur la base du travail de terrain que des enquêtes d'opinion, Bigot et Hoibian, 2010), mais aussi par une remise en question des modalités descendantes de l'action publique. Par conséquent, la mise en place des stratégies d'adaptation sur le territoire met moins l'accent sur un problème de connaissance ou d'éducation à l'environnement que sur des limites (et des opportunités de renouvellement) de l'action publique locale. Même si ces limites de l'appropriation sur le terrain de politiques descendantes ne sont pas le seul fait des pouvoirs publics, elles relèvent pour une part de la faible reconnaissance de la diversité des dimensions sociales et humaines, et de la pluralité des rationalités et des intérêts locaux qui interviennent sur les territoires, et pour une autre part de la difficulté à fonder les stratégies d'adaptation en matière d'intérêt général. Concernant ce dernier point, il ne s'agit pas de souligner la lutte difficile qui vise à porter le discours le plus légitime qui ferait autorité (par exemple, par le biais d'un combat d'expertise et de contreexpertise), mais bien de renverser la perspective et d'interroger ce qui fonde les principes légitimes à même de guider l'action publique, la mise en place et l'appropriation des politiques sur le territoire. Un renouvellement de l'action publique consiste à pen-

8. Tiré des échanges d'une journée d'étude tenue à Pau le 14 décembre 2012 et associant chercheurs et techniciens des collectivités territoriales aquitaines sur l'adaptation au changement climatique. http://set.univpau.fr/live/cesar\#Valorisation (Consultation : 10.10.2013). 
ser la conformité à un intérêt général non pas déjà constitué, mais produit d'un exercice démocratique, articulant plusieurs échelles, et qui reflète les spécificités territoriales, humaines et sociales, des terrains concernés. Les premiers enseignements de recherche du programme CESAR en Aquitaine suggèrent en outre qu'il ne faut pas sous-estimer la capacité réflexive des acteurs locaux à penser collectivement l'intérêt général. L'agir (et le penser) sur les territoires peut prendre en compte des enjeux étrangers aux préoccupations locales, directes et immédiates (comme les engagements pluriels de l'association Bizi en font par exemple la preuve - http://www.bizimugi.eu/).

Cette réflexion préliminaire met l'accent sur les défis que pose l'application des stratégies locales d'adaptation au changement climatique, en particulier sur la reconnaissance de la pluralité des dimensions territoriales (humaines et sociales), ainsi que sur le positionnement difficile de ces stratégies par rapport à un intérêt général en recomposition alors que la problématique climatique s'impose de façon relativement inconditionnelle. Ce double défi tiré de la recherche sur les terrains aquitains définit les contours de deux grands enjeux contemporains, relatifs à la connaissance et de la question environnementale et à sa prise en charge :

- L'enjeu épistémologique consiste à élaborer une approche géographique de l'environnement qui ne suit pas la trajectoire descendante des politiques qui s'imposent sur les territoires locaux, mais qui considère et part de la complexité de la construction de ces territoires (Rebotier, 2012a). C'est dans ce processus conjoint de construction de l'espace et de la question environnementale que les problématiques, politiques et dispositifs liés à l'adaptation prennent leur sens et s'incarnent sur le terrain. Il n'est pas inutile de chercher à acquérir plus d'information, de moyens, de compétences, et de ressources, de tâcher de mieux connaître pour mieux agir, ni même de chercher à être plus convaincant dans la mise en place d'une pensée aménagiste descendante. Mais à ces postures dialectiques (opposant le défi technique au défi social) s'en ajoutent d'autres plus attentives à la complexité des facteurs qui donnent des sens, des formes, des options privilégiées ou encore un cadre conceptuel à la question environnementale sur le terrain. Parmi les postures qui ancrent dans les sociétés le regard sur l'environnement, on peut distinguer deux entrées. L'une se caractérise par une approche du sensible, de l'expérience des individus, voire de la dimension esthétique de l'inscription dans et avec le milieu. Cette entrée articule le sujet réflexif, ses représentations, ses goûts, ses pratiques, ses choix à des logiques plus larges de construction des territoires, ou des paysages (Luginbühl et Terrasson, 2013 ; Lolive et Soubeyran, 2007). L'autre entrée est plus marquée par l'attention accordée aux rapports sociaux, aux cadres de contraintes et aux champs d'opportunités à l'intérieur desquels individus et groupes sont amenés à faire des choix et à agir. Ces cadres, nécessairement dynamiques, ne déterminent en rien les sociétés dans leur milieu, mais président pour beaucoup aux caractéristiques des territoires ainsi produits (Smith et O'Keefe, 1980 ; Rebotier, 2012b ; Ekers et Loftus, 2013). L'une comme l'autre entrée met 
l'accent sur des mécanismes humains et sociaux complémentaires qui interviennent au premier chef dans les modalités d'application des stratégies d'adaptation sur le terrain, dès leur conceptualisation.

- L'enjeu politique consiste à traduire ce changement d'approche de l'environnement sur le terrain. D'une part, dans une perspective opérationnelle, il s'agit d'envisager des dispositifs plus ouverts et plus collaboratifs que ce qu'offre l'action publique descendante, et qui relaient la complexité des territoires sans laquelle il ne semble y avoir d'appropriation possible (Berdoulay et Soubeyran, 2012). En convoquant les principaux obstacles rapportés du terrain, comme la non-rencontre des intérêts locaux, la défiance quant à la capacité d'agir du politique, ou le sentiment d'une concertation biaisée voire même l'absence de débat public, souvent galvaudé et mobilisé comme caution légitime, il est possible d'envisager plusieurs chantiers de travail. Ces derniers pourraient notamment concerner la consultation pour l'élaboration des stratégies d'adaptation (Boy, 2010), la définition des objectifs ou des modalités concrètes de ces stratégies, ou encore des formes de suivi et d'actualisation par retour d'expérience (Chalas et Soubeyran, 2009). D'autre part, dans une perspective de recherche, l'enjeu politique de l'application de stratégies d'adaptation mieux territorialisées consiste aussi pour les sciences humaines et sociales à critiquer la notion d'adaptation, à la fois catégorie d'analyse et d'action. Il est essentiel de la déconstruire et de la replacer dans les enjeux épistémologiques et dans les impasses opérationnelles identifiées. Ainsi, les sciences sociales se doivent d'élargir le périmètre de la critique, non pas seulement pour dénoncer les passagers clandestins véhiculés par l'adaptation, ou son instrumentalisation. Il s'agit surtout de saisir les conditions et le contexte qui permettent de légitimer l'usage de la notion, d'en réduire la portée à certains domaines, à certaines priorités, à certains intérêts, et d'en masquer ou d'en négliger d'autres. En ce sens, la critique est aussi une pratique qui contribue à renseigner les limites de l'action, en faisant de l'évidence de l'agir une construction sociale dont il convient d'identifier les ressorts.

\section{Bibliographie}

Agglomération Côte Basque - Adour (2012) Rapport développement durable. Bilan 2011, perspectives 2012. http://www.agglocotebasque.net/fileadmin/user_upload/fichiers/menu_pratique/rapport_MDD2011Ve rsion_web.pdf (Consultation : 10.10.2013).

Beck, U. (2003) La société du risque. Sur la voie d'une autre modernité. Paris, Flammarion.
Berdoulay, V. (2009) Possibilism. Dans Kitchin, R. et Thrift, N. (eds) International encyclopedia of human geography, Oxford, Elsevier, p. 312-321.

Berdoulay, V. et Soubeyran, O. (2012) Adaptation au changement climatique et pratiques réflexives en aménagement. Espace géographique, 41 (2), 169-180. 
Berdoulay, V. et Soubeyran, O. (eds) (2000) Milieu, colonisation et développement durable : perspectives géographiques sur l'aménagement. Paris, L'Harmattan.

Bertrand, F. et Larrue, C. (2007) Gestion territoriale du changement climatique. Une analyse à partir des politiques régionales. Programme Gestion et impacts du changement climatique (GICC2): ONERC, 64 p. http://www.gip-ecofor.org/doc/drupal/ gicc/Rapport Final Bertrand Larrue - synthese.pdf (Consultation : 10.10.2013).

Bertrand, F. et Rocher, L. (2011) L'intégration du changement climatique dans l'action publique locale, facteur de renouvellement du développement urbain durable ? Dans Béal, V., Gauthier, M. et Pinson, G. (eds) Le développement durable changera-t-il la ville? Le regard des sciences sociales, SaintEtienne, PUSE, p. 385-403.

Biesbroek, G.R., Swart, R.J., Carter, T.R., Cowan, C., Henrichs, T., Mela, H., Morecroft, M.D. et Rey, D. (2010) Europe adapts to climate change: Comparing National Adaptation Strategies. Global Environmental Change, 20 (3), 440-450.

Bigot, R. et Hoibian, S. (2010) Les Français avancent à grands pas sur la longue route écologique. Paris, CREDOC.

Blanc, N. et Lolive, J. (eds) (2007) Esthétique et espace public. Paris, Apogée.

Bloch, E. (1976) Le principe espérance. Paris, Gallimard.

Boy, D. (2010) Le Grenelle de l'environnement : une novation politique? Revue Française d'Administration Publique, 134 (2), 313324.

Callon, M., Lascoumes, P. et Barthe, Y. (2001) Agir dans un monde incertain. Essai sur la démocratie technique. Paris, Seuil.

Chalas, Y. et Soubeyran, O. (2009) Incertitude, environnement et aménagement. Quelle rupture? Dans Chalas, Y., Gilbert, C. et Vinck, D. (eds) Comment les acteurs s'arrangent avec l'incertitude? Paris, Editions des archives contemporaines, p. 135-157.

COBAS (2012) Plan Climat Energie Territorial de la COBAS. http://www.agglo-cobas.fr/ images/upload/pcet_valid_29032012.pdf (Consultation : 10.10.2013).

Demeritt, D. (2001) The construction of global warming and the politics of science. $A n$ nals of the Association of American Geographers, 91 (2), 307-337.

Ekers, M. et Loftus, A. (2013) Revitalizing the production of nature thesis: A Gramscian turn? Progress in Human Geography, 37 (2), 234-252.

Evrard, E. (2009) L'irrigation pastorale dans les Pyrénées: retour vers le futur? Mémoire de Master de Géographie, Université de Pau et des Pays de l'Adour.

Ferry, L. (1992) Le nouvel ordre écologique, l'arbre, l'animal et l'homme. Paris, Grasset.

Gilbert, C. (2003) Risques collectifs et situations de crise. Apports de la recherche en sciences bumaines et sociales. Paris, L'Harmattan.

Innerarity, D. (2008) Le futur et ses ennemis : De la confiscation de l'avenir à l'espérance politique. Paris, Climats.

Lolive, J. et Soubeyran, O. (eds) (2007) L'émergence des cosmopolitiques. Paris, La Découverte.

Luginbühl, Y. et Terrasson, D. (eds) (2013) Paysages et développement durable. Versailles, Quae.

Morin, E. (1993) Terre-Patrie. Paris, Seuil.

Rebotier, J. (2012a) Vulnerability Conditions and Risk Representations in Latin-America: Framing the territorializing urban risk. Global Environmental Change, 22 (2), 391-398. 
Rebotier, J. (2012b) La production de l'environnement. Colloque international Géographie, écologie, politique : un climat de changement. Communication orale, Orléans, 6-8 septembre.

Rist, G. (1996) Le développement, histoire d'une croyance occidentale. Paris, Presses de la Fondation Nationale de Sciences Politiques.

Salvestroni, P. (2012) La place de l'adaptation dans les politiques environnementales des collectivités locales. Quelles réactions face à l'urgence du changement climatique? Mémoire de Master en Géographie, Université de Pau et des Pays de l'Adour.

Serres, M. (1990) Le contrat naturel. Paris, François Bourin.
Smith, N. et O'Keefe, P. (1980) Geography, Marx and the concept of nature. Antipode, 12 (2), 30-39.

Theys, J. (2003) La gouvernance entre innovation et impuissance. Le cas de l'environnement. Développement durable et territoires, 2. http://developpementdurable.revues. org/1523 (Consultation : 10.10.2013).

While, A., Jonas, A. E.G. et Gibbs, D. (2010) From sustainable development to carbon control: eco-state restructuring and the politics of urban and regional development. Transactions of the Institute of British Geographers, 35, 76-93. 